\title{
GERMINACIÓN Y DESARROLLO DE PLANTULAS DE Myroxylon balsamum (L.) Harms EN EL DEPARTAMENTO DE SUCRE
}

\author{
Germination and seedling growth of Myroxylon balsamum (L.) Harms \\ in the department of Sucre
}

Palabras clave: alta variabilidad, bálsamo de tolú, criptocotilar, germinación acumulada, heterogeneidad, plántulas.

Key words: high variability, balsam of Tolú, chriptocotilar, cumulative germination, heterogeneity, seedlings.

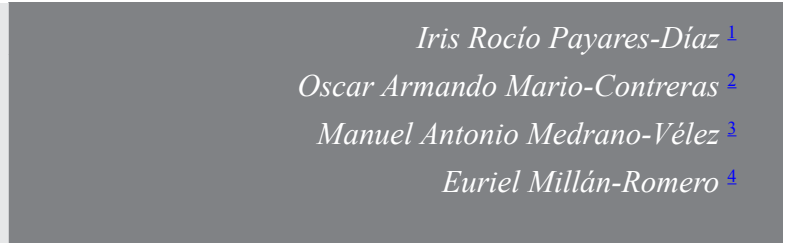

\section{RESUMEN}

Myroxylon balsamum tiene gran demanda en países de centro América gracias a sus propiedades botánicas y a las condiciones físicas de la madera, al ser materia prima en la industria farmacéutica, maderera y cosmética. En Colombia se encuentra casi amenazada (NT) por la presión al que ha sido sometido, ya sea por el uso de la resina, madera o por destrucción del hábitat, como sucede en el departamento de Sucre; lo que sugiere la importancia de realizar trabajos dirigidos a establecer el comportamiento de propagación que permita el restablecimiento poblacional en aéreas naturales. Este trabajo responde a esta necesidad, al cuantificar y analizar el comportamiento de germinación de M. balsamum.

Durante el proceso de germinación, la ruptura de la testa ocurrió 15 días posteriores a la siembra; obteniéndose un porcentaje de $77,2 \%$ y una tasa de germinación de 23.3 días. El coeficiente de variación para las variables altura de la plántula (AP), longitud de la raíz (LR), número de hojas
(NH) y número de nudos de la plántula $(\mathrm{NN})$ mostró en general una gran dispersión, pero esta fue mayor en las variables LR y NN. Lo anterior expone una alta heterogeneidad en el desarrollo de las plántulas, debido quizás a la procedencia de las semillas de plantas madres distintas, al grado de madurez de estas o a sus características genéticas y fisiológicas.

\begin{abstract}
Myroxylon balsamum is in great demand in Central America as its botanical and wood properties are useful in the pharmaceutical, timber and cosmetics industries. In Colombia, it is classified as near threatened (NT) due to over-exploitation for resin or wood, or habitat destruction in the department of Sucre. A guide was necessary to establish propagation practices for population restoration in natural areas. Here we quantify and analyze the germination behavior of M. balsamum. During the germination process, testa rupture occurred 15 days after sowing; obtaining a germination percentage of $77,2 \%$ and germination rate of 23.3 days. The
\end{abstract}

\footnotetext{
${ }^{1}$ Grupo de Investigación Botánica Neotropical. Universidad de Sucre. Sincelejo, Colombia Autor para correspondencia iris.payares@unisucre.edu.co.

${ }^{2}$ Universidad de Sucre. Sincelejo, Colombia.

${ }^{3}$ Universidad de Sucre. Sincelejo, Colombia.

${ }^{4}$ Grupo de investigación recurso Suelo-Agua. Universidad de Sucre. Sincelejo, Colombia. euriel.millan@unisucre.edu.co
} 
coefficient of variation for variable seedling height (AP), root length (LR), number of leaves (NH) and number of nodes per seedling $(\mathrm{NN})$ generally showed a wide dispersion, but it was greater in the variables LR y NN. This high behavioural diversity maybe due to the provenance of the seed by different mother plants, their maturity or differences in genetic and physiological characteristics.

\section{INTRODUCCIÓN}

El bálsamo de Tolú (Myroxylon balsamum) se encuentra distribuido desde el sur de México, pasando por Centroamérica hasta América del Sur (Berendsohn \& Araniva de González ,1989; Cordero et al., 2003), registrándose naturalmente en Argentina, Bolivia, Brasil, Colombia, Ecuador, Guyana, Paraguay, Perú, Surinam y Venezuela (Trópicos, 2013). Esta especie se encuentra en peligro de extinción y su aprovechamiento ha sido prohibido en Costa Rica (Cordero et al., 2003), pero en muchos de estos países no se conoce el estado real de su conservación.

En Colombia, según las categorías de la UICN (Unión Internacional para la Conservación de la Naturaleza) el Bálsamo fue categorizado como especie casi amenazada (NT) (Cárdenas \& Salinas, 2006), enfrentándolo a un deterioro poblacional a mediano plazo que lo ubicaría a las puertas de la extinción. En la Costa Caribe colombiana no se tiene registro sobre el grado de conservación de la especie, ni se han realizado trabajos dirigidos a establecerlo. De igual forma, en el departamento de Sucre sucede lo mismo, y dado un alto grado de intervención y degradación de grandes áreas para establecimiento de zonas agrícolas y pecuarias, es de esperarse que las poblaciones naturales hayan sido disminuidas drásticamente. Por ello, es importante realizar trabajos dirigidos a evaluar el potencial de propagación que tienen la especie, con miras a la reforestación.

En este sentido, referido a la propagación, la multiplicación vegetativa ha levantado los límites impuestos por largos ciclos de vida, fructificación $\mathrm{y} / \mathrm{o}$ floraciones irregulares y la alogamia, relegando a un segundo plano la reproducción sexual y facilitando la captura de genotipos individuales (Leakey et al., 1994). No obstante existen especies que no pueden ser propagadas vegetativamente con facilidad; en este sentido, la propagación vegetativa de Myroxylon balsamum, al utilizar reguladores de crecimiento como el Ácido Naftalen-acético (ANA) y Acido indol-butírico (AIB), no es óptima, M. balsamum presenta inhibidores del enraizamiento que afecta su capacidad para ser propagada vegetativamente (Mario \& Medrano, $\underline{2009}$ ). Por lo cual la germinación se convierte en una estrategia importante de propagación, con miras a la recuperación de poblaciones disminuidas o casi inexistentes.

El objetivo de este trabajo fue evaluar el comportamiento en la germinación y el subsecuente desarrollo de las plántulas de Myroxylon balsamum en invernáculo en el municipio de Sincelejo, Sucre.

\section{MATERIALES Y MÉTODOS}

El trabajo fue realizado en la ciudad de Sincelejo en el vivero "IPANEMA" a 9 $9^{\circ} 17$ '47" de latitud norte y $75^{\circ} 25$ ' 32 '”de longitud oeste; altitud 212 msnm y una pluviosidad media anual fue de 1580 $\mathrm{mm}$.

Las semillas se recolectaron de plantas madres de Bálsamo de Tolú (Myroxylum balsamum), ubicados en el corregimiento de la Garita municipio de Sincelejo, departamento de Sucre con coordenadas $9^{\circ} 11^{\prime} 68^{\prime \prime}$ de latitud norte y $75^{\circ} 22$ '30'de longitud oeste y con una altitud $192 \mathrm{msnm}$., se seleccionaron semillas de apariencia sana de aspecto liso sin rasgaduras. Las semillas colectadas se almacenaron a $5^{\circ} \mathrm{C}$ para evitar la desecación y muerte del embrión hasta el momento de la siembra, que ocurrió 2 días después de la recolección.

Antes de ser sembradas, fueron cortadas las secciones aladas y sometidas a un proceso de imbibición por 24 horas a través del cual se descartaron las semillas flotantes; se seleccionaron 250 semillas que fueron sembradas en bolsas de polietileno negras de $1 \mathrm{~kg}$ en un sustrato compuesto de arena, tierra negra y estiércol bovino en proporción 1:1:1; mezclado homogéneamente. La 
desinfección de este sustrato se realizó con solución de formaldehido al $0.4 \%$, cubriéndose con plástico oscuro durante 3 días y luego se saturó con agua de grifo, para eliminar los restos de formaldehido (Jarma et al., 2004).

La siembra fue realizada a $1-1,5 \mathrm{~cm}$. de profundidad y el riego de las mismas se realizó diariamente, a fin de garantizar una humedad adecuada (capacidad de campo) (Buitrago et al., 2004). Las bolsas de polietileno fueron mantenidas en un invernadero provisto de polisombra al $80 \%$, temperaturas entre $29^{\circ} \mathrm{C}$ a $34^{\circ} \mathrm{C}$, y humedad relativa promedio de $82 \%$.

La germinación de las semillas se cuantificó diariamente para determinar la tasa de germinación (TG) mediante la ecuación $\mathrm{TG}=(\mathrm{N} 1 \mathrm{~T} 1+\mathrm{N} 2 \mathrm{~T} 2+$ $\ldots .+\mathrm{NnTn}) /(\mathrm{N} 1+\mathrm{N} 2+\ldots .+\mathrm{Nn})$, donde $\mathrm{N}$ es el número de semillas germinadas no acumuladas $\mathrm{y}$ T es el tiempo en días (Hartmann \& Kester, 2001) y el porcentaje de germinación (PG) se calculó mediante la ecuación $\mathrm{PG}=$ Número de semillas germinadas/número de semillas totales $* 100$.

La evaluación de las variables altura de la plántula (AP), longitud de la raíz (LR), número de hojas $(\mathrm{NH})$ y número de nudos por plántula $(\mathrm{NN})$ fue realizada a los 36 días después de la siembra de las semillas; para establecer la altura de las plántulas (AP) se realizó la medición desde la base del tallo hasta el ápice foliar y la longitud de la raíz (LR) desde la base del tallo hasta el ápice de la raíz principal.

El análisis estadístico se realizó utilizando el programa STATGRAPHISCS Plus para Windows, versión 5.1 Edición Profesional Copyright (C) 2000 Statistical Graphics Corp. Se realizó una distribución de frecuencias, complementado con un estudio estadístico descriptivo de resumen y el coeficiente de correlación de Spearman para correlacionar las variables evaluadas.

\section{RESULTADOS}

La ruptura de la testa y la emergencia del epicótilo por encima del suelo ocurrió 15 días después de la siembra, mostrando germinación hipogea y plántulas criptocotilar; el surgimiento de los primordios foliares se dio a partir de los 18 días de iniciado el ensayo, tiempo a partir del cual, el área foliar de las plántulas comenzó a aumentar considerablemente con respecto al tallo que mostró un crecimiento radial lento, al igual que el largo y grosor de la raíz (Figura 1).

El porcentaje de semillas germinadas 15 días después de haber iniciado el ensayo fue de $8.14 \%$, momento en que emergió el mayor grupo de semillas. Posteriormente a los 20 días germinó un

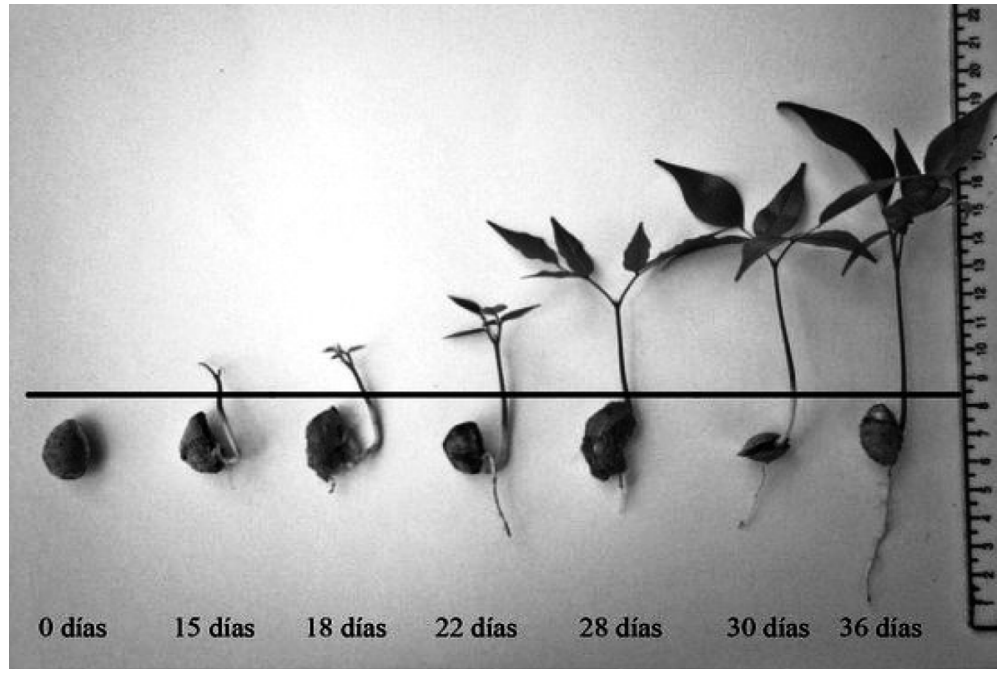

Figura 1. Germinación de semilla y crecimiento de plántulas de Myroxylon balsamum 
segundo grupo de semillas equivalente a un $7,75 \%$. A partir de ese momento, se aprecia un marcado declive seguido de un periodo irregular en las germinaciones hasta terminar a los 36 días (Tabla 1), con un porcentaje de germinación acumulada (PGA) de 77.2\% (Figura 2).

Tabla 1. Porcentaje de germinación diaria

\begin{tabular}{|c|c|c|}
\hline Días & Semillas geminadas & \% Germinación diaria \\
\hline 1 & 0 & 0 \\
\hline 2 & 0 & 0 \\
\hline 3 & 0 & 0 \\
\hline 4 & 0 & 0 \\
\hline 5 & 0 & 0 \\
\hline 6 & 0 & 0 \\
\hline 7 & 0 & 0 \\
\hline 8 & 0 & 0 \\
\hline 9 & 0 & 0 \\
\hline 10 & 0 & 0 \\
\hline 11 & 0 & 0 \\
\hline 12 & 0 & 0 \\
\hline 13 & 0 & 0 \\
\hline 14 & 0 & 0 \\
\hline 15 & 21 & 8.14 \\
\hline 16 & 18 & 6.98 \\
\hline 17 & 12 & 4.65 \\
\hline 18 & 15 & 5.81 \\
\hline 19 & 8 & 3.1 \\
\hline 20 & 20 & 7.75 \\
\hline 21 & 16 & 6.2 \\
\hline 22 & 10 & 3.88 \\
\hline 23 & 8 & 3.1 \\
\hline 24 & 9 & 3.49 \\
\hline 25 & 5 & 1.94 \\
\hline 26 & 3 & 1.16 \\
\hline 27 & 7 & 2.71 \\
\hline 28 & 6 & 2.33 \\
\hline 29 & 5 & 1.94 \\
\hline 30 & 5 & 1.94 \\
\hline 31 & 7 & 2.71 \\
\hline 32 & 3 & 1.16 \\
\hline 33 & 6 & 2.33 \\
\hline 34 & 5 & 1.94 \\
\hline 35 & 6 & 2.33 \\
\hline 36 & 3 & 1.16 \\
\hline Total & 192 & \\
\hline
\end{tabular}




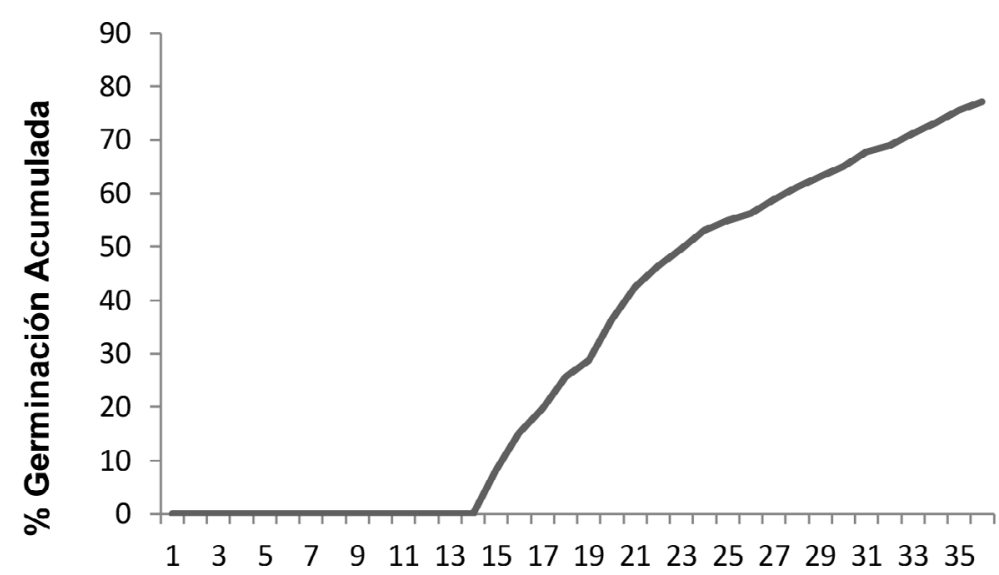

No. Días

Figura 2. Porcentaje de germinación acumulada

Las características morfológicas de las plántulas, altura de la planta (AP) y número de hojas $(\mathrm{NH})$ mostraron un coeficiente de variabilidad de 19.8 y $23.3 \%$, en cuanto a la longitud de la raíz (LR) y número de nudos $(\mathrm{NN})$ el coeficiente de variación fue de 42 y $32.1 \%$ respectivamente (Tabla 2).
Con respecto al coeficiente de correlación de Spearman, en el Tabla 3, se presentan los valores para las variable AP, LR, NH y NN, en plántulas de Myroxylon balsamum, para 36 días posteriores a la siembra.

Tabla 2. Análisis estadístico de resumen de las características morfológicas de las plántulas de Myroxylon balsamum, a los 36 días de la siembra

\begin{tabular}{lccccccc}
\hline Variables & D.E & C.V (\%) & $\begin{array}{c}\text { Valor } \\
\text { mínimo }\end{array}$ & $\begin{array}{c}\text { Valor } \\
\text { máximo }\end{array}$ & Rango & Media & Mediana \\
\hline $\begin{array}{l}\text { Altura de } \\
\text { la plántula } \\
\text { (cm) (AP) }\end{array}$ & 1.9 & 19.8 & 2.5 & 14 & 11.5 & 9.6 & 9.0 \\
$\begin{array}{l}\text { Longitud } \\
\text { de raíz } \\
\text { (cm) (LR) }\end{array}$ & 2.27 & 42.0 & 2 & 15 & 13 & 5.4 & 5.0 \\
$\begin{array}{l}\text { Número de } \\
\text { hojas (NH) } \\
\text { Número de } \\
\text { nudos (NN) }\end{array}$ & 0.7 & 23.3 & 2 & 7 & 5 & 3 & 3.0 \\
\hline
\end{tabular}

Tabla 3.Coeficiente de correlación de Spearman entre las variables AP $(\mathrm{cm}), \mathrm{LR}(\mathrm{cm}), \mathrm{NH}$ y NN, en plántulas de Myroxylon balsamum, 36 días posteriores a la siembra

\begin{tabular}{llll}
\hline & Largo de la raíz & Número de Hojas & Número de Nudos \\
\hline Altura de la planta & 0.127 & $0.275\left({ }^{* *}\right)$ & $0.213(* *)$ \\
& 0.078 & & 0.003 \\
& & & \\
Largo de la raíz & & $0.15\left(^{*}\right)$ & 0.113 \\
& & 0.032 & 0.120 \\
Número de hojas & & & $0.627\left(^{* *}\right)$ \\
\hline
\end{tabular}

Cada celda muestra el coeficiente de correlación. ** Altamente significativa $(\mathrm{p}<0.01)$ 


\section{DISCUSIÓN}

El inicio en el tiempo de la germinación de las semillas que comenzaron a germinar a los 15 días, coincide con lo señalado por Limongi et al. (2012); de otra parte, las diferencias en el inicio de la germinación del grupo total de semillas puede estar relacionado con las condiciones genéticas, morfológicas y fisiológicas individuales de cada una de ellas (Barbour et al., 1999; Baskin \& Baskin, 2001; Pérez, 2013), no todas maduraron al mismo tiempo y fueron obtenidas de plantas madres distintas, lo que implica que estos factores han intervenido a nivel de la respuesta germinativa de las mismas. Además, las altas temperaturas de hasta $34^{\circ} \mathrm{Cregistradas}$ durante varios días en la zona pudo haber influido también en el incremento de la velocidad de las reacciones fisiológicas y acelerar el proceso de germinación en unas semillas más que en otras (Hartmann \& Kester, 2001; PerozoBravo et al., 2006; Rodríguez, 2008). De otra parte, según Faccini \& Puricelli, (2006); Rodríguez, (2008); Gil \& Miranda, (2008) y Funes et al., (2009) las semillas poseen un rango de temperatura óptimo que afecta positivamente la germinacón, pero los cambios de temperaturas pueden provocar la pérdida progresiva de la viavilidad y disminuir el porcentaje de germinación; lo que significa que deben realizarse trabajos tendientes a determinar cómo afecta la temperatura la germinación de Myroxylon balsamum y su rango óptimo de temperatura.

El porcentaje de germinación acumulada (PGA) fue levemente superior a lo reportado por Cordero et al. (2003), quienes reportan un 60\% a $75 \%$ de germinación en viveros, y un $80 \%$ de germinación bajo la copa de los árboles; aunque Limongi et al. (2012) señalan que el porcentaje de germinación puede ser de $95 \%$ cuando las semillas son sembradas inmediatamente después de la cosecha. En el presente trabajo, pese a que las semillas fueron sembradas 2 días después de la recolección el porcentaje de germinación no se acercó a este valor, lo que puede evidenciar la influencia de factores fisiológicos como la falta de madurez del embrión, morfológicos como el tamaño de la semillas, genéticos propios de cada semilla o ambientales. Pero en general, el P GA en nuestro trabajo es alto y apoyado por lo encontrado por Cordero et al. (2003) y Limongi et al. (2012), Myroxylon balsamum posee un alto potencial de propagación para realizar proyectos de reforestación dirigidos a la restauración de áreas naturales en nuestro departamento.

Con respecto al coeficiente de variabilidad de las características morfológicas de las plántulas AP y NH (Tabla 2), se observa que estas variables son heterogéneas, pero muestran cierta cercanía entre sí, por lo que se podría pensar que existe relación entre altura de la planta y número de hojas, lo que coincide con lo encontado por Perozo-Bravo et al. (2006). En cuanto a la longitud de la raíz LR y número de nudos NN (Tabla 2) el coeficiente de variación indica que las variables son muy heterogéneas al existir un alto grado de dispersión. Al analizar el coeficiente de variación de todas las variables, se observa que la menos heterogénea es AP y la que muestra una mayor dispersión de sus valores es LR, sería lógico esperar que a mayor altura de la planta mayor longitud de la raíz, lo que no ocurrió, contrastando este resultado a lo hallado por Perozo-Bravo et al. (2006) en Anacardium occidentale L. La heterogeneidad en el largo de la raíz se puede explicar debido a que existen factores que pueden influir en su longitud, tales como, factores fisiológicos, genéticos que determinan su morfología (Smucker, 1993), edáficos que afectan el crecimiento de las raíces (Smart et al. 2006; Serra-Stepke \& Carey, 2010), de disponibilidad de elementos nutricionales minerales, como la nutrición carbonada y la intensidad lumínica (Aguirrezábal, et al, 2001), entre otros. Para los valores de rango los más altos fueron para LR y AP, esto puede estar relacionado con la diferencia en la emergencia de las plántulas; y para el caso de la mediana, esta fue similar a la media lo que deja ver una tendencia normal de las variables estudiadas.

Con respecto a al grado de significancia existió una correlación altamente significativa $(\mathrm{p}<0.01)$ entre las variables $\mathrm{NN}$ y NH, correspondiente a $(r=0.627)$, entre las variables AP respecto a la variable $\mathrm{NN}(\mathrm{r}=0.213)$ y AP y $\mathrm{NH}(\mathrm{r}=0.275)$ 
(Tabla 3). Naturalmente, existe una relación directamente proporcional de las variables AP, $\mathrm{NN}$ y $\mathrm{NH}$, es de esperarse que a mayor altura mayor número de nudos y por ende de hojas, similar a lo encontrado por Perozo-Bravo et al. (2006) en Anacardium occidentale L.

En general, existe una alta variabilidad en las plántulas de Myroxylon balsamum, lo que puede estar asociado con la procedencia de las semillas y el grado de madurez de estas que se reflejará en la disparidad de los tiempos de germinación o con aspectos genéticos, fisiológicos, edáficos y ambientales, como temperatura, que afecta porcentaje de germinación, tasa de absorción de agua, transporte de sustancias de reserva y la velocidad de las reacciones enzimática (Probert, 2000; Funes et al., 2009; Soto et al., 2010), humedad relativa, relacionada complejamente con la temperatura y afectando la cinética de la dormancia del embrión (Bazin et al., 2011); y luz, que fomenta la germinación en muchas semillas mediante la participación de fitocromos (Franklin \& Quail, 2010). Pero pese a los factores que puedan estar incidiendo tanto en la germinación como en el desarrollo de plantulas, las diferencias en el coeficiente de variación indican una alta variabilidad genética, lo que permitirá establecer poblaciones capaces de adaptarse a condiciones climáticas cambiantes y a un mayor flujo genético para resistir a enfermedades y presiones ambientales, lo que es apoyado por Fofana et al. (2009). Mientras que Young et al. (1996) exponen que cuando se fragmenta el hábitat y disminuyen los individuos de una población, se puede reducir el flujo genético $\mathrm{y}$ ocurren procesos de deriva genética $\mathrm{y}$ endogamia, tales procesos se pueden reducir al aumentar la variabilidad genética utilizando semillas de diferentes plantas madres.

\section{CONCLUSIONES}

El porcentaje de germinación acumulada (PGA) fue de $77.2 \%$, este valor es relativamente alto $\mathrm{y}$ proporciona un método de propagación óptimo para la repoblación de áreas naturales fragmentadas o destruidas. La heterogeneidad existente en las plántulas de Myroxylon balsamum, puede estar relacionada con la procedencia de distintas plantas madres, el grado de madurez que afectará el tiempo de germinación o por condiciones genéticas y fisiológicas propias de cada semilla. El coeficiente de variación de las variables altura de la planta (AP), longitud de la raíz (LR), número de hojas $(\mathrm{NH})$, y número de nudos de la plántula (NN) evidencian una alta variabilidad genética en Myroxylon balsamum, lo que es coherente con la procedencia de distintas plantas madres, pese a la influencia de factores bióticos y abióticos que puedan estar incidiendo sobre la germinación y desarrollo de las plántulas.

\section{REFERENCIAS BIBLIOGRÁFICAS}

Aguirrezábal, L.A.N., Orioli, G.A., Hernández, L.F., Pereyra V.R. \& Miravé, J.P. (2001). Girasol. Aspectos fisiológicos que determinan el rendimiento. Mar del Plata: Unidad Integrada Balcarce y Facultad de Ciencias Agrarias de la Universidad de Mar del Plata. 111 p.

Barbour, M.G., Burk, J.H., Pitts, W.D., Gillian, F.S., \& Schwartz, M.W. (1999). Allocation and life history patterns. En: Terrestrial plant ecology. Third edition. Benjamin Cummings. An imprint of Adison Wesley Longman. Icn. 88-116 p.

Baskin, C. C., y J. M., Baskin. (2001). Ecology, Biogeography and Evolution of Dormancy and Germination. San Diego: Academic Press. $667 \mathrm{p}$.

Bazin, J., Batlla, D., Dussert, S., El-MaaroufBouteau, H. \& Bailly, C. (2011). Role of relative humidity, temperature, and water status in dormancy alleviation of sunflower seeds during dry after-ripening. Journal of Experimental Botany, 62 (2), 627-640.

Berendsohn, W. G. y A. E. Araniva de González. (1989). Listado básico de la Flora Salvadorensis: Familia 118: Leguminosae. Cuscatlania, 1 (2), 1-16. 
Buitrago, N., Ramírez, M., Gómez, A., Rivero, A. \& Perozo, A. (2004). Efecto del almacenamiento de las semillas y la condición de luz postsiembra sobre la germinación y algunas características morfológicas de plantas de níspero (Manilkara zapota (Van Royen) (Jacq) Gill) a nivel de vivero. Revista de la Facultad de Agronomía, 21 (4), 344-353.

Cárdenas, D. \& Salinas, N. (2006). Libro Rojo de plantas de Colombia. Especies maderables amenazadas. I Parte. Bogotá: Instituto Amazónico de Investigaciones Científicas SINCHI. Ministerio de ambiente, Vivienda y Desarrollo Territorial. 169 p.

Cordero, J., Boshier, D.H., Barrance, A., Beer. J., Boshier, D.H., Chamberlain, J., Cordero, J., Detlefsen, G., Finegan, B., Galloway, G., Gómez, M., Gordon, J., Hands, M., Hellin, J., Hughes, C., Ibrahim, M., Kass, D., Leakey, R.; Mesén, F., Montero, M., Rivas, C., Somarriba, E., Stewart, J. \& Pennington, T. (2003). Árboles de Centroamérica: un manual para extensionistas. Oxford: OFI/CATIE.1079 p.

Faccini, D. \& Puricelli, E. (2006). Efecto de la temperatura y de la luz sobre la germinación de Nicotiana longiflora Cavaniles y Oenothera indecora Camb. Agriscientia, 23 (1), 15-21.

Fofana, I., Ofori, D., Poitel, M. \& Verhaegen, D. (2009). Diversity and genetic structure of teak (Tectona grandis L.f) in its natural range using DNA microsatellite markers. New Forests, 37 (1),175-195.

Franklin,K.A.\& Quail,P.H. (2010). Phytochrome functions in Arabidopsis development. Journal of Experimental Botany, 61 (1), $11-24$.

Funes, G.,Díaz， S. \&Venier, P. (2009). La temperatura como principal determinante de la germinación en especies del Chaco seco de Argentina. Ecología Austral, 19,129-138.

Gil, A. I. \& Miranda, D. (2008). Efecto de la temperatura, inmersión en agua $\mathrm{y}$ concentración de fitorreguladores sobre la germinación de semillas de papaya (Carica papaya L.). Revista Colombiana de ciencias hortícolas, 2 (1). 9-20.

Hartmann, H. \& F. Kester. (2001). Propagación de plantas, principios y prácticas. México: Editorial Continental. 760 p.

Jarma, A., Combatt, E., Polo, J. \& Beltran, J. (2004). (Recopilación). Propagación por miniestacas de roble (Tabebuia rosea) en función del sustrato y el regulador de crecimiento. Cordoba: Centro de investigaciones de la universidad de Córdoba.

Perozo-Bravo, A.,Ramírez-Villalobos M., Gómez-Degraves, A. \& BuitragoRueda, N. (2006). Germinación y caracterización morfológica de plántulas de merey (Anacardium occidentale L.) tipo Amarillo. Revista de la Facultade de Agronomía, 23 (1), 17-27.

Leakey, R., Newton, A. C. \& Dick, J. (1994). Capture of genetic variation by vegetative propagation: processes determining success. EN: Oberschelp, G. P. J. y Marcó, M. A. 2010. Efecto del ácido 3-indolbutírico sobre el enraizamiento adventicio y la altura de plantines clónales de Prosopis alba Grisebach. Quebracho, $18(1,2), 112-119$.

Limongi, R., Guiracocha, G. \& Nieto, E. (2012). Bálsamo: Myroxylon spp. Especie de uso múltiple del bosque seco del Ecuador. EstaciónExperimentalPortoviejo, Estación experimental del Litoral Sur. Programa Nacional de Forestaría. Boletín técnico No 152. INIAP-MAGAP-SENESCYT. Guayaquil: Editorial Grafiservi. 20 p. 
Mario, O. A. \& Medrano, M. A. (2009). Evaluación del potencial de propagación de la especie promisoria Myroxylon bálsamum, por germinación de semillas $\mathrm{y}$ estimulación hormonal de estacas usando dos fitoreguladores "ANA" Y "AIB" en el departamento de Sucre. Sincelejo: Universidad de Sucre.

Pérez, S.B. (2013). Viviparidad, Germinación y Supervivencia en Stenocereus thurberi (Cactaceae). (Tesis. Maestría en Ciencias). México: Colegio de Posgraduados. Institución de Enseñanza e investigación en Ciencias Agricola.Montecillo. 92p

Probert, R. (2000). The role of temperature in the regulation of seed dormancy and germination. 261-292 p. En: Fenner, M (ed.) Seeds: The Ecology of Regeneration in Plant Communities. CAB International, Wallingford, UK.

Rodríguez, M. R. (2008). Influencia de la temperatura en la germinación de semillas de Caesalpinia spinosa (Molina) Kuntze "taya" de cuatro localidades del Departamento La Libertad, Perú. Arnaldoa, $15(1), 87-100$.
Serra-Stepke, I y Carey, V. (2010). Sistema radical de la vid: importancia y principales factores que lo afectan. Ciencia Ahora, 25, 69-79.

Smart, D.R., Schwass, E., Lakso, A. \& Morano, L. (2006). Grapevine rooting patterns: a comprehensive analysis and a review. American Journal of Enology and Viticulture, 57 (1), 89-104.

Smucker, A.J.M. (1993). Soil environmental modifications of root dynamics and measurement. The Annual Review of Phytopathology, 31, 191-216.

Soto, J. L., Valiengo, S. \& César de Paula, R. (2010). Germinación de semillas de Albizia hassleri a diferentes temperaturas, en condiciones de laboratorio. Bosque, 31 (1), 39-44.

Tropicos. (2013). Myroxylon bálsamum. Missouri Botanical Garden. Recuperado de http://www.tropicos.org/ Name/13003351?tab=distribution

Young, A.G., Boyle, T. \& Brown, T. (1996). The population genetic consequences of habitat fragmentation for plants. Trends in Ecology \& Evolution, 11. 413-418. 